\title{
Elastic and Viscoelastic Characterization of Mouse Oocytes Using Micropipette Indentation
}

\author{
XINYU Liu, ${ }^{1} \mathrm{~J}_{\mathrm{IAYI}} \mathrm{SHI},{ }^{2}$ Zong Zong, ${ }^{2} \mathrm{KaI}-\mathrm{TaK} \mathrm{WAN}^{2}$ and Yu Sun ${ }^{3}$ \\ ${ }^{1}$ Department of Mechanical Engineering, McGill University, Montreal, QC, Canada; ${ }^{2}$ Department of Mechanical and Industrial \\ Engineering, Northeastern University, Boston, MA, USA; and ${ }^{3}$ Department of Mechanical and Industrial Engineering and \\ Institute of Biomaterials and Biomedical Engineering, University of Toronto, Toronto, ON, Canada
}

(Received 23 February 2012; accepted 14 May 2012; published online 30 May 2012)

Associate Editor Tingrui Pan oversaw the review of this article.

\begin{abstract}
This paper reports the first quantitative comparison study of elastic and viscoelastic properties of oocytes from young and aged mice. A force measurement technique, including a poly(dimethylsiloxane) (PDMS) cell holding device and a sub-pixel computer vision tracking algorithm, is utilized for measuring forces applied to an oocyte and resultant cell deformations in real time during oocyte manipulation. To characterize elastic and viscoelastic properties of the oocytes, a stress-relaxation indentation test is performed. A two-step, large-deformation mechanical model is developed to extract the mechanical properties of the oocytes from the measured force-deformation data. The experimental results demonstrate that the aged oocytes are significantly softer (instantaneous modulus: $2.2 \mathrm{vs} .5 .2 \mathrm{kPa}$ in young oocytes) but more viscous (relaxation time: 4.1 vs. $2.3 \mathrm{~s}$ in young oocytes) than the young oocytes.
\end{abstract}

Keywords-Stress-relaxation test, PDMS device, Cell force measurement, Large deformation, Young's modulus, Viscoelastic properties, Aged mouse, BioMEMS, Cell mechanics.

\section{INTRODUCTION}

Characterizing mechanical properties of biological cells is crucial in understanding cellular structures and physiological functions, predicting cellular responses to mechanical cues and stimuli, and correlating cell mechanical properties with pathophysiological conditions. In embryology and reproduction research,

Address correspondence to Xinyu Liu, Department of Mechanical Engineering, McGill University, Montreal, QC, Canada, Kai-Tak Wan, Department of Mechanical and Industrial Engineering, Northeastern University, Boston, MA, USA, and Yu Sun, Department of Mechanical and Industrial Engineering and Institute of Biomaterials and Biomedical Engineering, University of Toronto, Toronto, ON, Canada. Electronic mail: xinyu.liu@mcgill.ca, ktwan@coe.neu.edu, sun@mie.utoronto.ca mechanical characterization of mammalian oocytes represents a potential technique to detect cellular defects in pre-implantation oocytes, ${ }^{12}$ and to assess quality and bio-viability of processes such as cryopreservation. ${ }^{7}$

Assisted reproduction technologies require the reproductive quality of oocytes to be evaluated to select the healthy ones for in vitro fertilization (IVF) use. Existing techniques for oocyte quality assessment include morphology analysis, ${ }^{25,27}$ genetic screening, $, 5,29$ spectroscopy-based metabolomic profiling, ${ }^{22,28,33}$ and polscope-based spindle imaging. ${ }^{20,24}$ These techniques have the following limitations: (i) morphological analysis is often subjective and fails to provide definitive prediction for oocyte quality; (ii) genetic screening employs a deoxyribonucleic acid (DNA) sampling procedure that may impair the oocyte and result in lower development competence ${ }^{18}$; and (iii) metabolomic profiling and spindle imaging demand specific analysis equipment and usually involve complex spectral data analysis. The development of effective and low-cost approaches for oocyte quality evaluation can potentially benefit both fundamental reproductive research and clinical IVF practices.

We recently developed a vision-based force measurement technique, ${ }^{13,15}$ which includes a poly(dimethylsiloxane) (PDMS) cell holding device and a sub-pixel computer vision tracking algorithm, to measure the applied mechanical forces and the resultant cell deformations simultaneously, during oocyte manipulation such as intracytoplasmic sperm injection (ICSI). Different from the existing techniques for measuring forces applied to a cell (e.g., micropipette aspiration, ${ }^{16,26}$ AFM indentation, ${ }^{2}$ magnetic bead measurement, ${ }^{1}$ optical tweezers, ${ }^{3}$ and MEMS-based measurement ${ }^{9,30}$ ), the new technique can be readily integrated into a microinjection system (Fig. 1), and the measurement of 


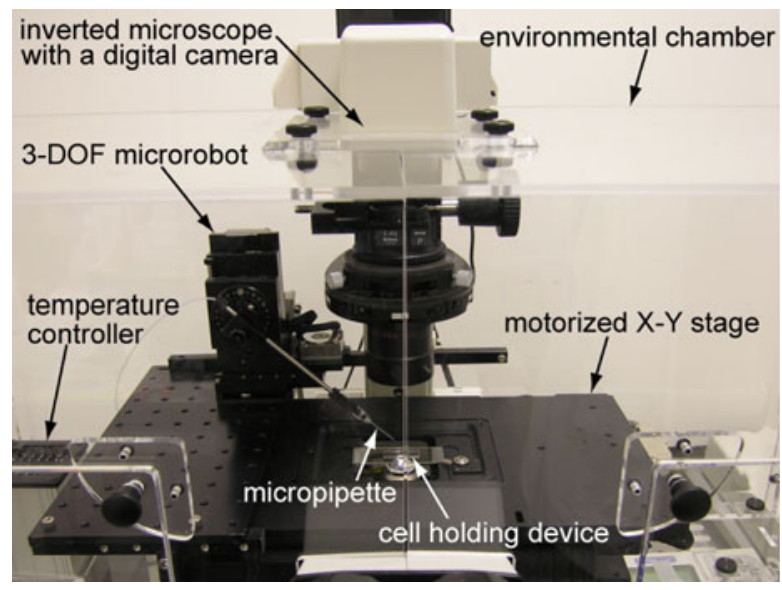

FIGURE 1. Mouse oocyte microinjection system used for force measurement.

force and deformation is carried out during microinjection (in situ), without any separate characterization accessories and procedures. Our preliminary mechanical deformation measurement showed distinct mechanical characteristics of healthy (young) and aging-induced defective (aged) mouse oocytes as a result of structural changes in cytoskeleton and the glycoprotein shell of zona pellucida (ZP). ${ }^{12}$

Up to date, there are no comparative studies reported in the literature on elastic and viscoelastic properties of young and aged oocytes. In order to quantitatively reveal the mechanical differences between young and aged mouse oocytes, this study performed stress-relaxation indentation tests of mouse oocytes using the vision-based force measurement technique. By fitting the experimental data to a twostep, large-deformation mechanical model, we report the elastic and viscoelastic properties of the young and aged mouse oocytes for the first time. These mechanical property data of healthy and defective oocytes could potentially lead to quantitative guidelines for oocyte quality evaluation, and in the meanwhile, spark further studies to investigate the pathways between the oocyte defects and their manifestation in cell mechanical properties. This work was partially presented at the 2011 International Conference on Miniaturized Systems for Chemistry and Life Sciences. ${ }^{14}$ In the present paper, technical details and comparison data between computational and experimental results are presented.

\section{MATERIALS AND METHODS}

\section{Mouse Oocyte Preparation}

Oocytes are harvested from young (8-12 weeks old) imprinting-control-region (ICR) female mice at their fertile age, and aged (40-45 weeks old) counterparts that are approaching the end of their reproductive lifespan. Previous studies suggest that the aged oocytes and their embryos are compromised in terms of developmental competence as a result of multiple cellular defects such as meiotic irregularities and mitochondrial dysfunction. ${ }^{6}$

All experiments are conducted in compliance with federal laws and institutional guidelines, and are approved by the Mount Sinai Hospital Animal Care Committee in Toronto. Young and aged ICR mice are superovulated by intraperitoneal injection with $5 \mathrm{IU}$ of pregnant mare's serum gonadotropin (PMSG) (Sigma) and $48 \mathrm{~h}$ later with $5 \mathrm{IU}$ of human chorionic gonadotropin (hCG) (Sigma). Oocytes are collected at $16 \mathrm{~h}$ post-hCG and cultured in potassium simplex optimization medium (KSOM, Specialty Media). The dimensions of the young and aged oocytes were similar with an average diameter of $90 \mu \mathrm{m}$.

\section{Experimental Setup}

Figure 1 shows a microinjection system for in situ measurement of mechanical load applied to an oocyte. It consists of a PDMS cell holding device, an inverted microscope (TE2000-S, Nikon) with a CMOS digital camera (A601f, Basler), a three-degree-of-freedom (3-DOF) micromanipulator (MP-285, Sutter) for motion control of the injection micropipette, a motorized $\mathrm{X}-\mathrm{Y}$ stage (ProScan II, Prior) for positioning oocytes, and a temperature-controlled chamber (Solent Scientific) to maintain oocytes at $37^{\circ} \mathrm{C}$.

\section{Vision-Based Force Measurement Technique}

The vision-based force measurement technique is based on the PDMS device (Figs. 2a and 2b) and a subpixel visual tracking algorithm, which are used together to resolve the applied forces with nanonewton resolution during microinjection. The force measurement is realized by visually tracking elastic deformations of low-stiffness structures in the cell holding device, and subsequently, transforming material deformations into forces. Details of this technique were reported previously. ${ }^{13,15}$ In this study, we utilized this technique for stress-relaxation indentation tests of mouse oocytes to quantify their elastic and viscoelastic properties.

Figure $2 \mathrm{~b}$ shows the PDMS device that integrates an array of cavities (180 $\mu \mathrm{m}$ in diameter) for containing individual mouse oocytes. Inside each cavity, lowstiffness posts - with height and diameter of $H=$ $45 \mu \mathrm{m}$ and $D=12 \mu \mathrm{m}$, respectively — are arranged in a circular pattern, acting as a cell trap and providing a mechanical support for the oocyte during microinjection. The PDMS device was constructed using standard soft lithography. ${ }^{15}$ 
(a)

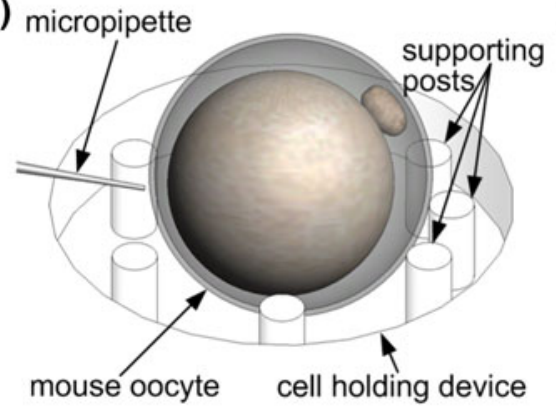

(b)

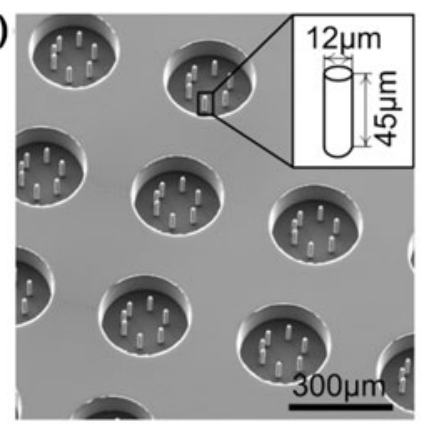

(c)

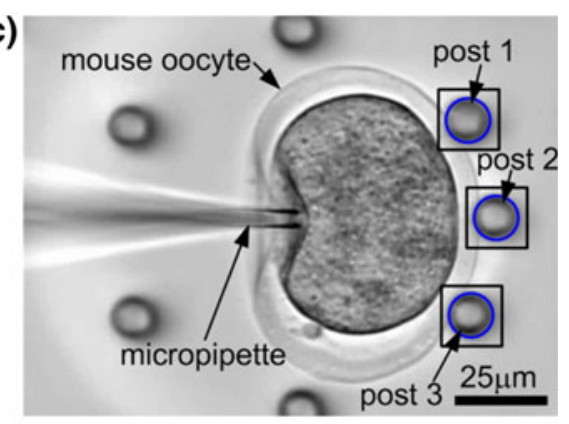

(d)

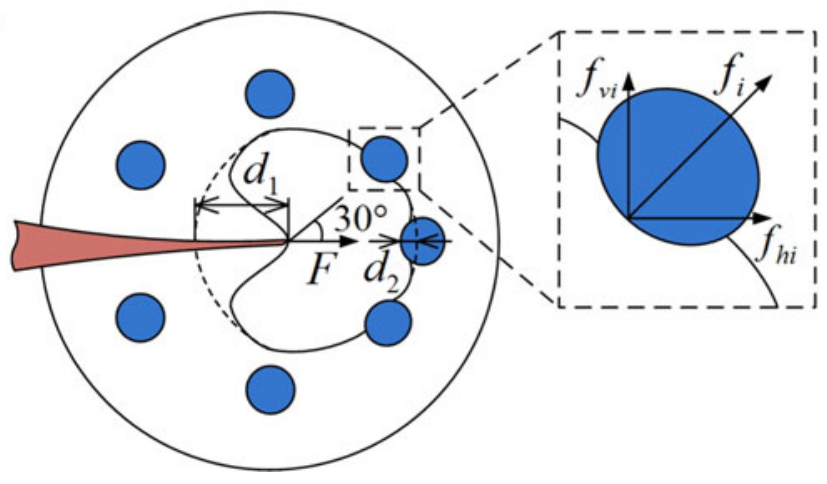

(e)

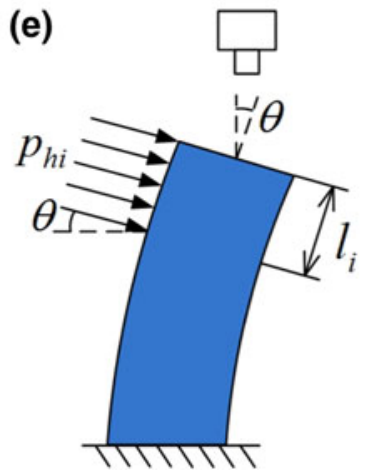

FIGURE 2. Vision-based force measurement of mouse oocytes. (a) Schematic of the working principle. (b) SEM image of a PDMS cell holding device. (c) Indentation forces deform the mouse oocyte and deflect three supporting posts. Post deflections were measured by visually tracking the displacements of the post top circles (blue). (d) Force balance on the cell under indentation. (e) Post deflection model.

The working principle is outlined as follows. While the micromanipulator drives a sperm-carrying micropipette to indent an oocyte, and the applied force, $F$, is transmitted via the cell body to the three supporting posts on the leeside (Fig. 2c), causing them to deflect elastically (Fig. 2e). At equilibrium, $F$ is balanced by the horizontal components, $f_{\text {hi }}$, of the transmitted force on the posts (Fig. 2d). A sub-pixel visual tracking algorithm measures the post deflections at $30 \mathrm{~Hz}$. The actual force on each post can be found using the forcedeflection relation of the post derived from linear elasticity. The viscoelastic effect of the PDMS posts was safely ignored as previous studies demonstrated that the error caused by treating a PDMS micropost elastically instead of viscoelastically is within $10 \% .{ }^{11}$ The PDMS post, with a height-to-diameter ratio smaller than 5, was treated as a Timoshenko beam, and both bending and shearing of the post were considered. ${ }^{32}$ The indentation force applied by the micropipette is:

$$
F=\sum_{i=1}^{3} \frac{d_{i} l_{i}}{\frac{40 l_{i}(1+\gamma)\left(2 H-l_{i}\right)}{9 \pi E D^{2}}+\frac{8\left(l_{i}^{4}+8 H^{3} l_{i}-6 H^{2} l_{i}^{2}\right)}{3 \pi E D^{4}}}
$$

where $i=1,2,3$ denotes the post numbers, $d_{i}$ is the horizontal deflection, $l_{i}$ is the contact length between the oocyte and the supporting posts (Fig. 2e), $H$ and $D$ are post height and diameter; $E$ and $\gamma$ are Young's modulus and Poisson's ratio $\left(\gamma=0.5\right.$ for $\left.\operatorname{PDMS}^{17}\right)$. Using nanoindentation, elastic modulus of the cell holding device was measured to be $524.7 \pm 22.1 \mathrm{kPa}$ $(n=5)$.

A constant indentation speed of $60 \mu \mathrm{m} / \mathrm{s}$ was used in all measurements. The cell-post contact was recorded in situ using a side-view microscope (Navitar 1-50487AD; 20× objective) with a digital camera (A601f, Basler) at $30 \mathrm{~Hz}$, and its setup is illustrated in Fig. S1 (Supplemental Material). The contact length, $l_{i}$, was measured offline using Image ${ }^{\circledR}{ }^{\circledR}$. Time-dependent behavior was observed such that $l_{i}=v_{i} t$, where $t$ is time and $v_{i}$ is the increasing speed of $l_{i}$, which was measured to be in the range of $v_{i}=0.8-1.2 \mu \mathrm{m} / \mathrm{s}$. Fortunately, the $v_{i}$-dependence of Eq. (1) is fairly insignificant. In fact, the variation of $F$ is confined to merely $1 \%$ within the range of $v_{i}$. The average speed of $1.0 \mu \mathrm{m} / \mathrm{s}$ was therefore used to calculate injection forces for all the oocytes.

The horizontal deformation of an indented oocyte consists of two components: (i) a global deformation induced by indentation at the contact location of the micropipette tip ( $d_{1}$ in Fig. $2 \mathrm{~d}$ ), and (ii) a local deformation at the contact location of post 2 ( $d_{2}$ in Fig. $\left.2 d\right)$. The deformation, $d_{1}$, was determined by subtracting 
the deflection of post 2 (Fig. 2c) from the displacement of the micropipette.

\section{Stress-Relaxation Indentation Test}

In order to quantify both elastic and viscoelastic properties of the mouse oocytes, we conducted stressrelaxation indentation test. As shown in Fig. 3a, the micromanipulator controlled the micropipette to indent an oocyte at a constant speed of $60 \mu \mathrm{m} / \mathrm{s}$ (stage I in Fig. 3a), stopped at a displacement of $40 \mu \mathrm{m}$, and then held the micropipette's position for $45 \mathrm{~s}$ (stage II in Fig. 3a), during which the indentation force, as schematically shown in Fig. 3b, was measured by visually tracking the deflections of the supporting posts. While holding the micropipette's position, the local deformation, $d_{2}$, increased due to the effect of cellular relaxation; however, this relaxation-induced change of $d_{2}$ can be safely ignored due to the fact that $d_{1} \gg d_{2}$ (Fig. 3), and the total cell deformation, $d$, can thus be regarded as a constant value. The force-time data obtained during cell relaxation thus indicate viscoelastic properties of the oocyte.

\section{Mechanical Model for Oocyte Deformation}

A mechanical model was developed to account for large deformation of the oocytes and extract their
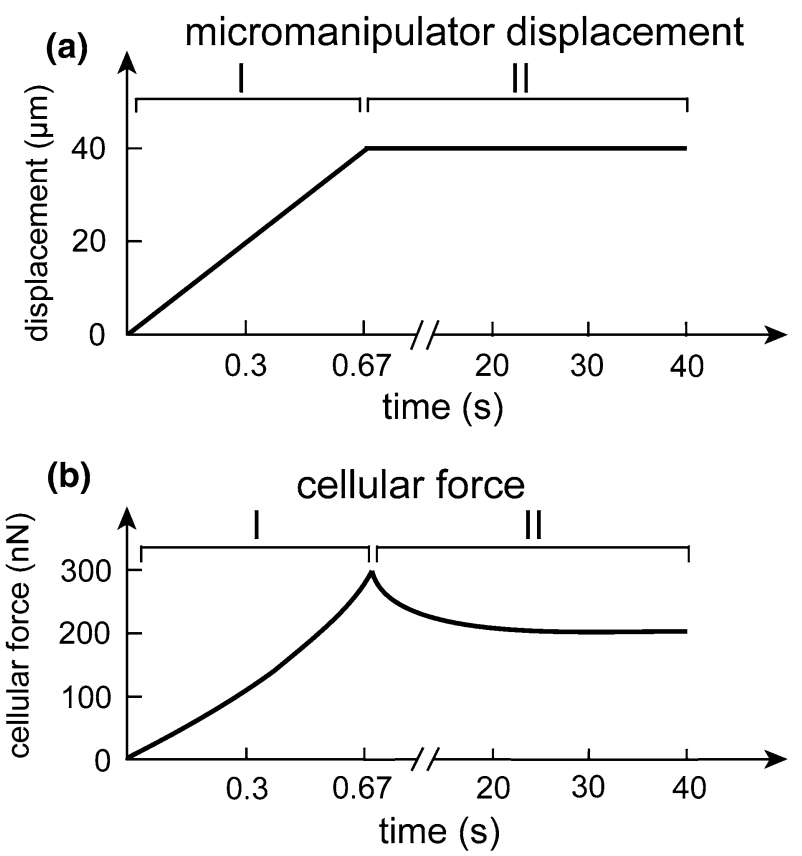

FIGURE 3. Stress-relaxation indentation test. (a) Displacement of the micromanipulator as a function of time. The micromanipulator controlled the micropipette to indent the oocyte at a constant speed of $60 \mu \mathrm{m} / \mathrm{s}$ (stage I), and maintained a vertical displacement of $40 \mu \mathrm{m}$ for $45 \mathrm{~s}$ (stage II). (b) Schematic of the expected force response as a function of time. elastic and viscoelastic properties. Oocytes are known to comprise a thick ZP encapsulating a biorheological liquid of cytoplasm. In the presence of an external load, both the ZP and cytoplasm deform simultaneously and interact with one another to reach a mechanical equilibrium. Here, we focus on the mechanical behavior of the overall cell, rather than the detailed oocyte microstructures. We modeled the oocyte as an isotropic and continuum sphere. In the first attempt, the constitutive relation and deformed profile of the oocyte were computed by finite element analysis (FEA) with large deformation using ABAQUS (Standard version 6.9-2). However, FEA did not lead to a converged solution because of the large deformation as the indentation depth approaching $40 \%$ of the sphere diameter.

We then pursued an alternative semi-analyticalnumerical model as an approximation to estimate the elastic and viscoelastic properties of the mouse oocytes, where the cell deformation is described by two consecutive steps as shown in Fig. 4a. At Step 1, the central load $F$ is theoretically replaced by the compression of a rigid plate perpendicular to the loading axis with the same force magnitude $F$. Mechanical equilibrium is attained when the resulting approach distance reaches $y_{1}$, creating a planar contact circle with radius, $c$. At Step 2, the top plate is substituted by a central load with the same magnitude, $F$, distributed over the micropipette tip. The original planar region (with a radius of $c$ ) was treated as an infinite half continuum and the micropipette a cylindrical punch indenter with radius, $a$, thus forming an indentation dimple with depth, $y_{2}$. The overall displacement of the micropipette tip is $y=y_{1}+y_{2}$.

The constitutive relation in Step 1, $F(y)$ when $y<y_{1}$, was obtained by large-deformation FEA. The oocyte was modeled as an incompressible, isotropic, axisymmetric, and continuum sphere with an elastic modulus, $E$, and Poisson's ratio, $\gamma=0.50$. Three boundary conditions were considered: (i) the three confining posts in the leeside are modeled as rigid cylinders; (ii) the nodes at the axisymmetric axis have zero translational displacements along $x$ and $z$ directions, and zero rotational displacement about $y$ direction $\left(U_{x}=U_{z}=U R_{y}=0\right.$ in Fig. 4b); and (iii) contacts between the oocyte and the posts are frictionless. The stress-displacement analysis was performed using an 8-node linear brick, reduced integration elements (C3D8R). To promote computational efficiency, the density of elements was set to the highest at the contact regions, and diminishes further away. Figures $4 \mathrm{~b}$ and $4 \mathrm{c}$ illustrate a $3 \mathrm{D}$ meshed model of the oocyte and a typical deform-stress field after simulation, respectively. The deformation profile of an oocyte, generated by the FE model, was also compared with the experimental results for model validation (Fig. 5). 
(a)

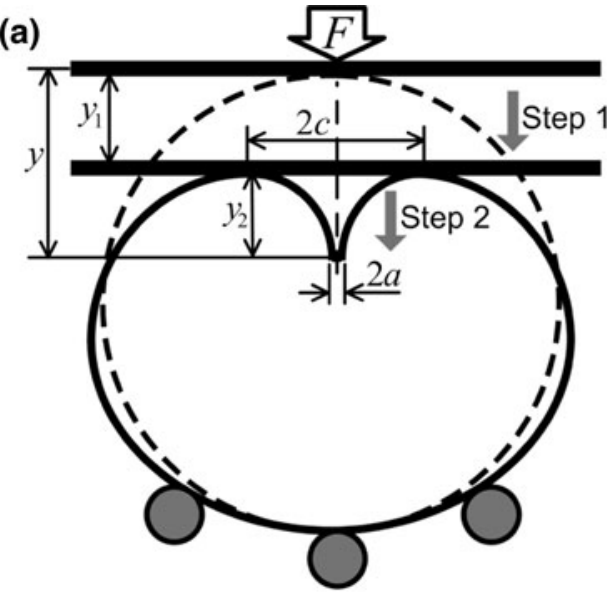

(c)

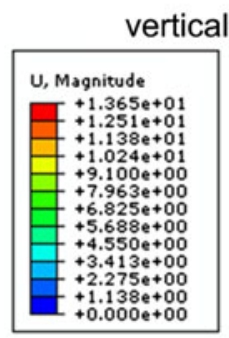

(b)

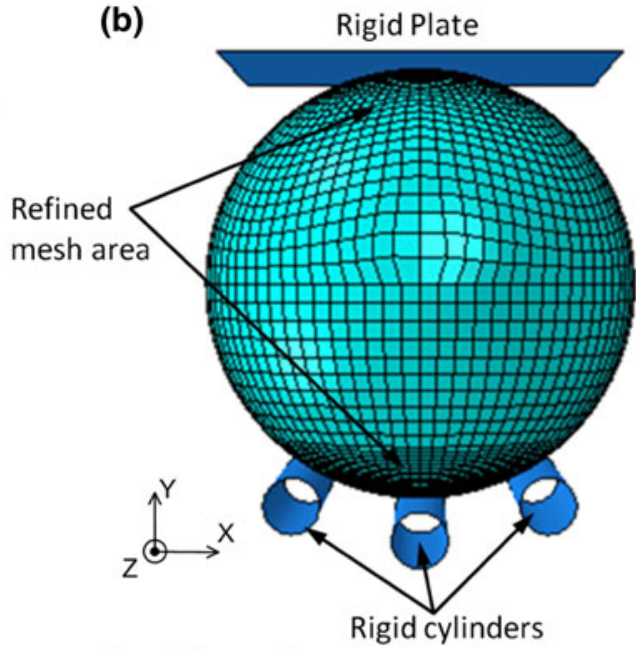

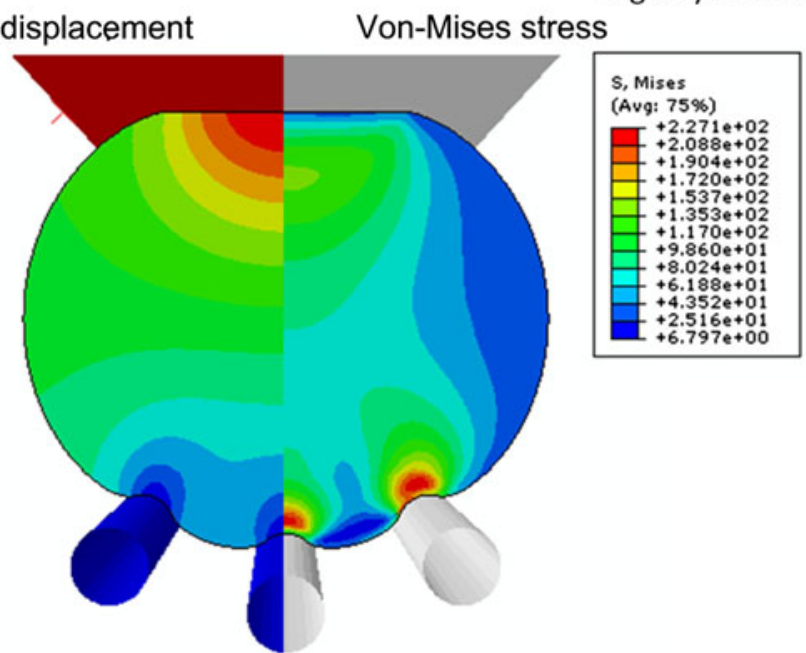

FIGURE 4. A two-step, large-deformation model of the oocyte. (a) Schematic diagram of the two-step deformation, including an initial plate compression (Step 1) and a subsequent flat-punch indentation with the half-space assumption (Step 2). (b) A meshed 3D model of the oocyte for FEA. (c) The distribution of displacement (left) and Von-Mises stress (right) in an oocyte after compression.

A classical elastic model that describes the indentation of an infinite half space by a cylindrical flat-end punch (with radius, $a$ ) is given by Maugis ${ }^{19}$

$$
y_{2}=\frac{1-v^{2} F}{2 E} \frac{F}{a}
$$

provided $a$ is much smaller than the contact radius, $c$, in Step 1, or $a \ll c$. The overall constitutive relation, $F(y)$, was therefore obtained from the two-step deformation model, and fitted to the experimental data to extract the cell's elastic modulus. In the two-step loading process, the superposition principle of elastic theory was applied. The infinite half-space boundary condition was used for simplicity of the analytical model in Step 2. As shown later in the "Results" section, the proposed two-step model interpreted the experimental data with good agreement.

To account for the viscoelastic behavior, the elastic model is slightly modified. During relaxation, the external load decreases as a function of time though the deformed profile does not show any measurable change. It is therefore assumed that only the Young's modulus decreases while all other parameters remain constant. The viscoelastic behavior of the oocytes was described by a standard viscoelastic model with three parameters, including instantaneous modulus $E_{0}$, relaxed modulus $E_{\mathrm{R}}$, and relaxation time $\tau$. To obtain these parameters, the apparent elastic modulus as a function of time during relaxation was first calculated, by fitting the force-time data (stage II in Fig. 3b) and the constant deformation $d$ (during relaxation) into the two-step model. The calculated time-dependent elastic modulus was then fitted into the viscoelastic model to extract the viscoelastic parameters of the oocytes.

$$
E(t)=\left(E_{0}-E_{\mathrm{R}}\right) \exp \left(-\frac{t}{\tau}\right)+E_{\mathrm{R}}
$$




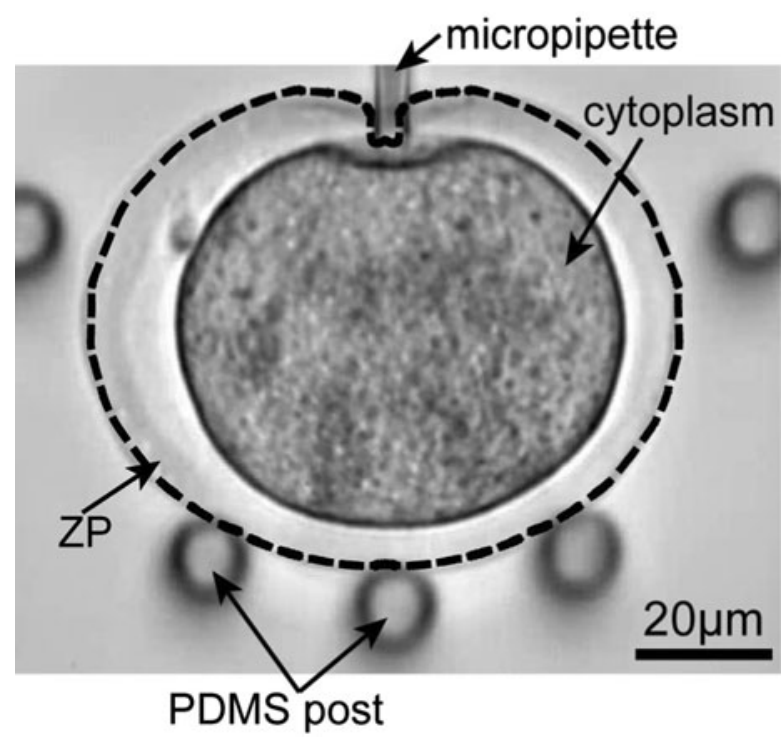

FIGURE 5. Comparison between experimental deformed profile and theoretical model (dash line).

where $E_{0}$ is the instantaneous modulus at $t=0, E_{\mathrm{R}}$ is the relaxed modulus at $t \rightarrow \infty$, and $\tau$ is the relaxation time.

\section{Statistical Analysis}

Experimental data are analyzed using Student's $t$-test (SigmaStat 3.5, Systat Software Inc.). The error bars represent one standard deviation from the mean value.

\section{RESULTS}

All the experiments were performed at $37{ }^{\circ} \mathrm{C}$ inside the environmental chamber. With a $40 \times$ objective (NA $0.55)$, the pixel size of the imaging system was calibrated to be $0.24 \mu \mathrm{m} \times 0.24 \mu \mathrm{m}$. With the current optical system, the tracking resolution of the post deflection was determined to be $0.12 \mu \mathrm{m}(0.5$ pixel $)$ and the force measurement resolution $2 \mathrm{nN}$. Micropipette tips used for indenting mouse oocytes were $1.8 \mu \mathrm{m}$ in diameter.

Stress-relaxation indentation test were performed on eight young oocytes and eight old oocytes, and the time-dependent indentation force was recorded (stages I and II in Fig. 3b). Figure 6a shows the force-deformation data of the young (blue, $n=8$ ) and old (red, $n=8$ ) oocytes during indentation. The elastic properties of the indented oocytes were extracted by fitting the theoretical model to individual set of force-deformation data (the solid line in Fig. 6a). The elastic modulus of young oocytes was found to be $3.1 \pm 0.4 \mathrm{kPa}$, which is approximately twice that of aged oocytes $(1.6 \pm 0.4 \mathrm{kPa})$.

Figure $6 \mathrm{~b}$ shows the force-time measurements during the relaxation process (stage II in Fig. 3b) for both
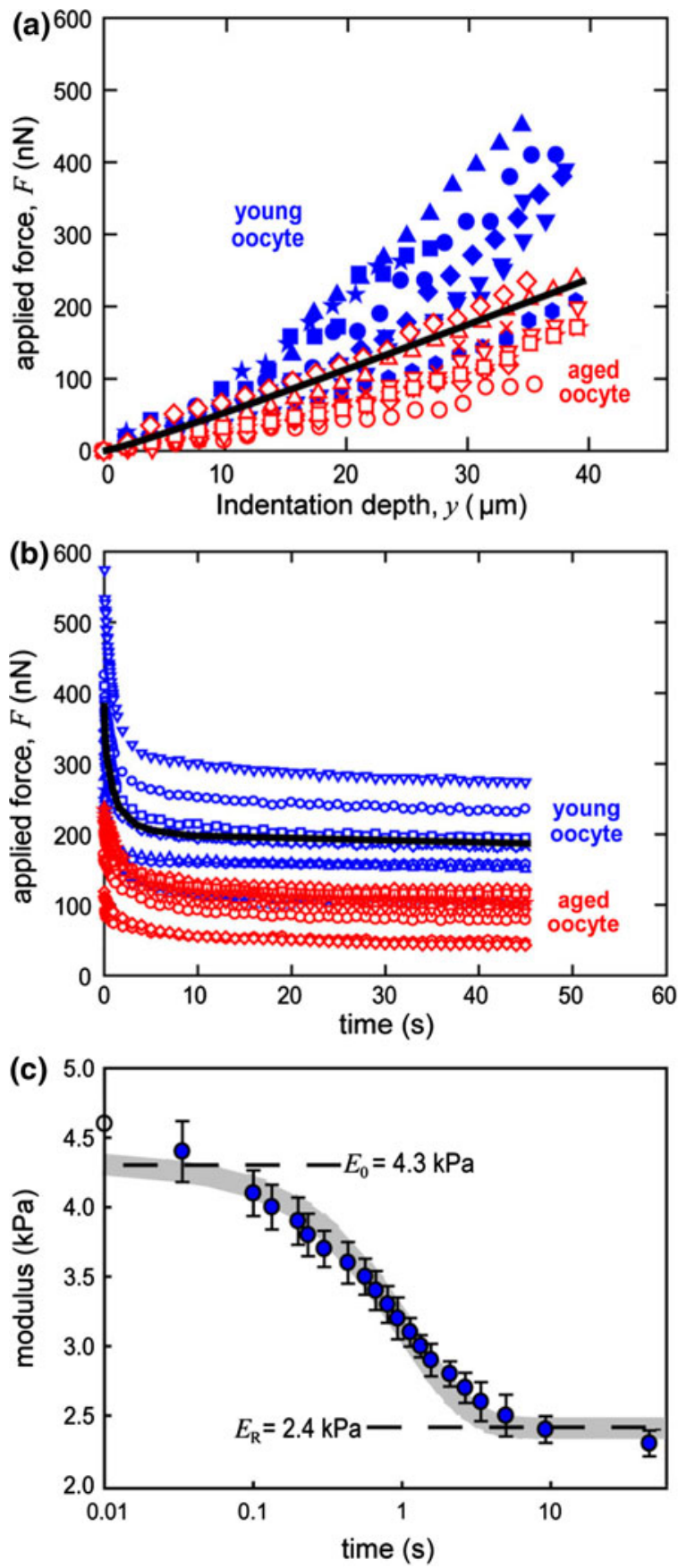

FIGURE 6. Experimental results of the stress-relaxation test of young and aged oocytes. (a) Force-deformation data from the young (black) and aged (red) oocytes at Stage I. (b) Forcetime data from the young (black) and aged (red) oocytes at Stage II. The black lines are typical curve fits to single sets of experimental data. (c) Experimental results of apparent elastic modulus as a function of time for a sample young oocyte.

young and aged oocytes and the theoretical curve fit. Figure $6 \mathrm{c}$ shows the time-dependent modulus and the curve fit according to Eq. (3). The viscoelastic parameters of young and old oocytes are summarized in Table 1 . We found that the moduli of aged oocytes, $E_{0}$ and $E_{\mathrm{R}}$, are about half of these of young oocytes, 
TABLE 1. Summary of elastic and viscoelastic properties of young $(n=8)$ and aged $(n=8)$ oocytes.

\begin{tabular}{lcccc}
\hline & Elastic property & \multicolumn{2}{c}{ Viscoelastic properties } \\
\cline { 3 - 5 } Oocyte type & Young's modulus $E(\mathrm{kPa})$ & $\begin{array}{c}\text { Relaxed } \\
\text { modulus } E_{0}(\mathrm{kPa})\end{array}$ & modulus $E_{\mathrm{R}}(\mathrm{kPa})$ & $\begin{array}{c}\text { Relaxation } \\
\text { time } \tau(\mathrm{s})\end{array}$ \\
\hline Young oocytes & $3.1 \pm 0.4$ & $5.2 \pm 0.5$ & $2.7 \pm 0.4$ & $2.3 \pm 0.2$ \\
Aged oocytes & $1.6 \pm 0.4^{*}$ & $2.2 \pm 0.6^{*}$ & $1.0 \pm 0.3^{*}$ & $4.0 \pm 0.6^{*}$ \\
\hline
\end{tabular}

${ }^{*} p<0.001$.

while the relaxation time of aged oocytes, $\tau$, is approximately twice that of young oocytes. These results indicate that the aged oocytes are significantly softer but more viscous than the young oocytes.

\section{DISCUSSION}

This study provides quantitative measurement of the elastic and viscoelastic properties of young and aged mouse oocytes. Direct comparison of the results to other studies is not available since most of the previous research on mouse oocytes or embryos has been focused on the mechanical properties of $\mathrm{ZP}$, which is the glycoprotein membrane enveloping the oocyte. The reported elastic modulus of the ZP, which was measured by various experimental techniques and mechanical models, ${ }^{8,21,30}$ ranges from 8.26 to $17.9 \mathrm{kPa}$. Using the micropipette aspiration technique, the Young's modulus of the human oocyte $\mathrm{ZP}$ was measured to be $2.41 \pm 0.75 \mathrm{kPa}^{23}$ While the two step model used in this paper treat the oocyte as a solid sphere, the acquired elastic modulus results involve contributions from the moduli of both $\mathrm{ZP}$ and cytoplasm. It is well known that the cytoplasm has a much lower Young's modulus than that of the ZP, which contributes to the lower overall elastic modulus value of the mouse oocyte compared to the results in literature. ${ }^{23}$

There is no existing data in literature on viscoelastic properties of the mouse oocytes. Viscoelastic characterization of articular chondrocytes, ${ }^{4,10}$ performed via indentation techniques, reveals values of viscoelastic parameters $\left(E_{0}, E_{\mathrm{R}}\right.$ and $\left.\tau\right)$ at the same order of magnitude as the ones reported in this study. In addition, the infinite half-space assumption has been adopted in flat punch indentation for two-step modeling, when the flattened cell thickness is on the order of five times the inner radius of the pipette, ${ }^{26,31}$ with the reasoning that the smaller the inner radius of the pipette, the better the results of the half-space model. This requirement is fulfilled in this research considering the average diameter of the oocyte $(\sim 90 \mu \mathrm{m})$ is almost two orders of magnitude higher than the diameter of the indenter probe $(1.8 \mu \mathrm{m})$.

Our findings reveal that the measured elastic, instantaneous and relaxed moduli of young mouse oocytes are over one time higher than those of aged mouse oocytes. This result quantitatively demonstrates that the young and old mouse oocytes are mechanically different. The differences in mechanical properties are attributed to the differences in structure of the ZP and cytoskeleton. Our previous scanning electron microscopy (SEM) examination has shown that the surface of young oocytes $\mathrm{ZP}$ is a fibrous network with numerous pores, compared to a rough $\mathrm{ZP}$ surface deprived of pores in old oocytes. ${ }^{12}$ Transmission electron microscopy (TEM) imaging of the cross sections of ZP glycoprotein structures indicate the glycoproteins in young oocytes $\mathrm{ZP}$ are significantly denser than those in the ZP of old oocytes. ${ }^{12}$ The analysis of F-acting contents via fluorescent staining also shows that old oocytes contain much less F-actin in cytoplasm than young oocytes. ${ }^{12}$ This lack of glycoprotein in cell membrane and F-actin in cytoskeleton cause poor mechanical support for subjected mechanical loads, therefore decrease the overall mechanical properties in old oocytes.

In summary, this paper described the stress-relaxation indentation test of mouse oocytes using a visionbased force measurement technique, for characterizing their elastic and viscoelastic properties. A microfabricated PDMS device, integrated in a mouse oocyte microinjection system, was used for measuring forces applied to an oocyte and corresponding cellular deformations by visually tracking deflections of elastic supporting posts on the device. A two-step, largedeformation mechanical model was developed by combining analytical and finite element approaches. Through stress-relaxation tests, the elastic and viscoelastic properties of young and aged mouse oocytes was, for the first time, quantified and compared. Comparison results of the mechanical differences between young and aged mouse oocytes were correlated well to our previous findings on the structural changes of cell membrane and cytoskeleton in the aged oocytes.

\section{ELECTRONIC SUPPLEMENTARY MATERIAL}

The online version of this article (doi: 10.1007/s10439-012-0595-3) contains supplementary material, which is available to authorized users. 


\section{ACKNOWLEDGMENTS}

We thank Roxanne Fernandes, Andrea Jurisicova, and Robert F. Casper from Toronto Mount Sinai Hospital for their assistance on mouse oocyte preparation. XY is supported by the Natural Sciences and Engineering Research Council of Canada and the McGill University. YS is supported by the Natural Sciences and Engineering Research Council of Canada and the Canada Research Chairs Program. JS, ZZ and KTW are supported by the US National Science Foundation.

\section{REFERENCES}

${ }^{1}$ Bausch, A. R., F. Ziemann, A. A. Boulbitch, K. Jacobson, and E. Sackmann. Local measurements of viscoelastic parameters of adherent cell surfaces by magnetic bead microrheometry. Biophys. J. 75:2038-2049, 1998.

${ }^{2}$ Charras, G. T., P. P. Lehenkari, and M. A. Horton. Atomic force microscopy can be used to mechanically stimulate osteoblasts and evaluate cellular strain distributions. Ultramicroscopy 86:85-95, 2001.

${ }^{3}$ Dai, J. W., and M. P. Sheetz. Mechanical-properties of neuronal growth cone membranes studied by tether formation with laser optical tweezers. Biophys. J. 68:988-996, 1995.

${ }^{4}$ Darling, E. M., S. Zauscher, and F. Guilak. Viscoelastic properties of zonal articular chondrocytes measured by atomic force microscopy. Osteoarthr. Cartil. 14:571-579, 2006.

${ }^{5}$ Donoso, P., C. Staessen, B. C. J. M. Fauser, and P. Devroey. Current value of preimplantation genetic aneuploidy screening in IVF. Hum. Reprod. Update 13:15-25, 2007.

${ }^{6}$ Hamatani, T., G. Falco, H. Akutsu, C. A. Stagg, A. A. Sharov, D. B. Dudekula, V. VanBuren, and M. S. H. Ko. Age-associated alteration of gene expression patterns in mouse oocytes. Hum. Mol. Genet. 13:2263-2278, 2004.

${ }^{7}$ Jain, J. K., and R. J. Paulson. Oocyte cryopreservation. Fertil. Steril. 86:1037-1046, 2006.

${ }^{8}$ Khalilian, M., M. Navidbakhsh, M. R. Valojerdi, M. Chizari, and P. E. Yazdi. Estimating young's modulus of zona pellucida by micropipette aspiration in combination with theoretical models of ovum. J. R. Soc. Interface 7: 687-694, 2010.

${ }^{9}$ Kim, K. Y., X. Y. Liu, Y. Zhang, J. Cheng, X. Y. Wu, and Y. Sun. Elastic and viscoelastic characterization of microcapsules for drug delivery using a force-feedback MEMS microgripper. Biomed. Microdevices 11:421-427, 2009.

${ }^{10}$ Koay, E. J., A. C. Shieh, and K. A. Athanasiou. Creep indentation of single cells. J. Biomech. Eng. 125:334-341, 2003.

${ }^{11}$ Lin, I. K., K. S. Ou, Y. M. Liao, Y. Liu, K. S. Chen, and X. Zhang. Viscoelastic characterization and modeling of polymer transducers for biological applications. J. Microelectromech. Syst. 18:1087-1099, 2009.

${ }^{12}$ Liu, X. Y., R. Fernandes, A. Jurisicova, R. F. Casper, and Y. Sun. In situ mechanical characterization of mouse oocytes using a cell holding device. Lab Chip 10:2154-2161, 2010.
${ }^{13}$ Liu, X. Y., K. Kim, Y. Zhang, and Y. Sun. Nanonewton force sensing and control in microrobotic cell manipulation. Int. J. Robot. Res. 28:1065-1076, 2009.

${ }^{14}$ Liu, X. Y., J. Shi, Z. Zong, R. Fernandes, R. F. Casper, A. Jurisicova, K. T. Wan, and Y. Sun. Characterizing elastic and viscoelastic properties of young and aged mouse oocytes using a PDMS microdevice. International Conference on Miniaturized Systems for Chemistry and Life Sciences, pp. 467-469, 2011.

${ }^{15}$ Liu, X. Y., Y. Sun, W. H. Wang, and B. Lansdorp. Visionbased cellular force measurement using an elastic microfabricated device. J. Micromech. Microeng. 17:1281, 2007.

${ }^{16}$ Liu, X. Y., Y. F. Wang, and Y. Sun. Cell contour tracking and data synchronization for real-time, high-accuracy micropipette aspiration. IEEE Trans. Autom. Sci. Eng. 6:536-543, 2009.

${ }^{17}$ Mark, J. E. Polymer Data Handbook. Oxford, New York: Oxford University Press, 2009.

${ }^{18}$ Mastenbroek, S., M. Twisk, J. van Echten-Arends, B. Sikkema-Raddatz, J. C. Korevaar, H. R. Verhoeve, N. E. Vogel, E. G. Arts, J. W. de Vries, P. M. Bossuyt, C. H. Buys, M. J. Heineman, S. Repping, and F. van der Veen. In vitro fertilization with preimplantation genetic screening. N. Engl. J. Med. 357:9-17, 2007.

${ }^{19}$ Maugis, D. Contact, Adhesion, and Rupture of Elastic Solids. Berlin; New York: Springer, 2000.

${ }^{20}$ Moon, J. H., C. S. Hyun, S. W. Lee, W. Y. Son, S. H. Yoon, and J. H. Lim. Visualization of the metaphase II meiotic spindle in living human oocytes using the polscope enables the prediction of embryonic developmental competence after icsi. Hum. Reprod. 18:817-820, 2003.

${ }^{21}$ Murayama, Y., M. Yoshida, J. Mizuno, H. Nakamura, S. Inoue, Y. Watanabe, K. Akaishi, H. Inui, C. E. Constantinou, and S. Omata. Elasticity measurement of zona pellucida using a micro tactile sensor to evaluate embryo quality. J. Mamm. Ova Res. 25:8-16, 2008.

${ }^{22}$ Nagy, Z. P., S. Jones-Colon, P. Roos, L. Botros, E. Greco, J. Dasig, and B. Behr. Metabolomic assessment of oocyte viability. Reprod. Biomed. Online 18:219-225, 2009.

${ }^{23}$ Navidbakhsh, M., M. Khalilian, M. R. Valojerdi, M. Chizari, and P. E. Yazdi. Alteration in the mechanical properties of human ovum zona pellucida following fertilization: experimental and analytical studies. Exp. Mech. 51:175-182, 2011.

${ }^{24}$ Rienzi, L., F. Ubaldi, M. Iacobelli, M. G. Minasi, S. Romano, and E. Greco. Meiotic spindle visualization in living human oocytes. Reprod. Biomed. Online 10:192-198, 2005.

${ }^{25}$ Rienzi, L., F. Ubaldi, M. Iacobelli, S. Romano, M. G. Minasi, S. Ferrero, F. Sapienza, E. Baroni, and E. Greco. Significance of morphological attributes of the early embryo. Reprod. Biomed. Online 10:669-681, 2005.

${ }^{26}$ Sato, M., D. P. Theret, L. T. Wheeler, N. Ohshima, and R. M. Nerem. Application of the micropipette technique to the measurement of cultured porcine aortic endothelial cell viscoelastic properties. J. Biomech. Eng. 112:263-268, 1990.

${ }^{27}$ Scott, L., A. Finn, T. O'Leary, S. McLellan, and J. Hill. Morphologic parameters of early cleavage-stage embryos that correlate with fetal development and delivery: prospective and applied data for increased pregnancy rates. Hum. Reprod. 22:230-240, 2007.

${ }^{28}$ Scott, R., E. Seli, K. Miller, D. Sakkas, K. Scott, and D. H. Burns. Noninvasive metabolomic profiling of human embryo culture media using Raman spectroscopy predicts embryonic reproductive potential: a prospective blinded pilot study. Fertil. Steril. 90:77-83, 2008. 
${ }^{29}$ Sher, G., L. Keskintepe, M. Keskintepe, M. Ginsburg, G. Maassarani, T. Yakut, V. Baltaci, D. Kotze, and E. Unsal. Oocyte karyotyping by comparative genomic hybridization provides a highly reliable method for selecting "competent" embryos, markedly improving in vitro fertilization outcome: a multiphase study. Fertil. Steril. 87:1033-1040, 2007.

${ }^{30}$ Sun, Y., K. T. Wan, K. P. Roberts, J. C. Bischof, and B. J. Nelson. Mechanical property characterization of mouse zona pellucida. IEEE Trans. Nanobiosci. 2:279-286, 2003.

${ }^{31}$ Theret, D. P., M. J. Levesque, M. Sato, R. M. Nerem, and L. T. Wheeler. The application of a homogeneous half-space model in the analysis of endothelial cell micropipette measurements. J. Biomech. Eng. 110:190199, 1988.

${ }^{32}$ Ugural, A. C., and S. K. Fenster. Advanced Strength and Applied Elasticity. Upper Saddle River, NJ: Prentice Hall PTR, 2003.

${ }^{33}$ Vergouw, C. G., L. L. Botros, P. Roos, J. W. Lens, R. Schats, P. G. A. Hompes, D. H. Burns, and C. B. Lambalk. Metabolomic profiling by near-infrared spectroscopy as a tool to assess embryo viability: a novel, non-invasive method for embryo selection. Hum. Reprod. 23:1499-1504, 2008. 